\title{
Yeasts from the Bovine Rumen
}

\author{
By R. T. J. CLARKE \\ Plant Chemistry Division, Department of Scientific and \\ Industrial Research, Palmerston North, New Zealand \\ AND MARGARET E. DI MENNA \\ Soil Bureau, Department of Scientific and Industrial Research, \\ Wellington, New Zealand
}

(Received 12 November 1960)

\begin{abstract}
SUMMARY
Yeasts belonging to the genera Candida and Trichosporon have been isolated in small numbers from the rumen of fistulated cows. Similar yeasts were not found on samples of feed material. The yeasts present fell into two groups dependent on the diet of the cow from which they were isolated.
\end{abstract}

\section{INTRODUCTION}

The yeast flora of the lower intestinal tract of cattle was studied by Parle (1957) and by Van Uden \& do Carmo Sousa (1957); but in spite of the similarity of the rumen environment to that of the lower intestinal tract, rumen yeasts have received little attention. A rumen yeast flora has not been reported previously although the work of several authors has suggested its existence. Ingram \& McGaughey (1948) isolated 'Candida-like' organisms from the rumen of sheep and Cunningham \& Brisson (1955) noted an alcoholic fermentation in the stomachs of lambs on a high glucose diet. Lubinsky $(1957,1958)$ listed yeasts as food for many species of ciliate protozoa from the rumens of reindeer, sheep and goats. Rumen yeasts may not play an important part in the rumen fermentation, but from the veterinary point of view they may be of importance as a reservoir of infection. Yeast infections of the bovine udder are relatively frequent and have been caused by species of Candida, Trichosporon and Pichia. The present investigation into the rumen yeast flora was prompted by the isolation of Candida albicans from the rumen during a preliminary survey (Clarke, 1960).

\section{METHODS}

The rumen contents of five cows, one normal (no. 34) and four with rumen fistulas (no. 1, 90, 293, 294) were sampled at intervals between January 1959 and February 1960. All cows were fed hay during the winter months (April-August). During the spring and summer (September-February) all cows, with the exception of cow no. 1 which was hay-fed, were given fresh red clover (Trifolium pratense L.). As stall feeding could be carried out only on working days (Monday to Friday inclusive), the cows were turned out to pasture on Saturdays and Sundays. In spring and summer the pasture was predominantly red clover and in winter predominantly ryegrass (Lolium perenne $\mathbf{L}$.). 
Samples of rumen contents were removed from fistulated cows by an operator who wore sterile rubber gloves. The non-fistulated cow was sampled by using a sterile oesophageal tube, with suction.

The pasture, fresh clover and clover hay on which the cows were feeding were each sampled on two occasions. Hay samples were taken with aseptic precautions and clover and pasture samples were cut with sterile box shears.

All samples were plated on glucose peptone agar $(4 \%, w / v$, glucose; $1 \%, w / v$, peptone; $2 \%, w / v$, agar; adjusted to $\mathrm{pH} 4)$. Serial dilutions of the samples to be plated were made by the method of di Menna (1957). All cultures of rumen samples were incubated at $39^{\circ}$, but in addition two from hay-fed cows and parallel cultures from the non-fistulated cow were incubated at room temperature. Cultures of hay and pasture samples were incubated both at room temperature and at $39^{\circ}$. After 3-6 days of incubation all yeast-like colonies were picked off for identification from plates where the colonies were well separated.

Yeast cultures were maintained on the following medium $(\%, w / v):$ Malt Extract (Difco) 0.3; Yeast Extract (Difco) 0.3; Neopeptone (Difco) 0.2; glucose 1; in tap water. The yeasts obtained were identified by the criteria of Lodder \& Kreger-van Rij (1952).

\section{RESULTS}

\section{Rumen isolates}

Particulars of the rumen samples and of the yeasts isolated are given in Table 1. Nine species belonging to three genera were identified from the 134 isolates examined. Viable counts made during the isolation procedure ranged from 80 to 13,000 organisms/g. rumen contents. Particulars of the species isolated are given below.

Candida spp. Ten isolates of Candida albicans (Robin) Berkhout were recovered from two clover-fed cows and one from a hay-fed cow. Two of the isolates were tested for pathogenicity by intravenous inoculation into rabbits. Both killed rabbits within $48 \mathrm{hr}$. and were recoverable from the animals' kidneys. Four isolates of C. tropicalis (Cast.) Berkhout, four of C. krusei (Cast.) Berkhout and two of $C$. rugosa (Anderson) Diddens \& Lodder were recovered from two clover-fed cows. One isolate of $C$. rugosa was obtained from a hay-fed cow.

Trichosporon spp. Sixteen isolates of Trichosporon cutaneum (de Beurm., Gougerot $\&$ Vaucher) Ota were recovered from two cows, both fed on hay. These isolates resembled those of $\boldsymbol{T}$. cutaneum type II described by Lodder \& Kreger-van Rij (1952, p. 624) in that cultures on solid media were cream, dull, dry, becoming folded, but not hairy. They differed in that assimilation of maltose was not apparent on solid carbon-source auxanograms and was extremely weak in liquid carbon-source auxanograms. Cultures of two of these isolates were sent to Dr N. J. W. Kreger-van Rij (Yeast Division, Centraalbureau voor Schimmelcultures) who considered them to be variants of $T$. cutaneum.

Eighty-four isolations of Trichosporon sericeum (Stautz) Diddens \& Lodder were made from four cows, seventeen from a clover-fed cow and the rest from hay-fed cows. Twenty-six of the isolates were recovered from room temperature cultures. In the key to the genus Trichosporon Lodder \& Kreger-van Rij (1952) separate $T$. sericeum from $T$. capitatum Diddens \& Lodder by its reduced mycelium and relatively strong assimilation of galactose. The eighty-four strains isolated here produced mycelium readily under aerobic conditions but also assimilated galactose 
Yeasts from the bovine rumen

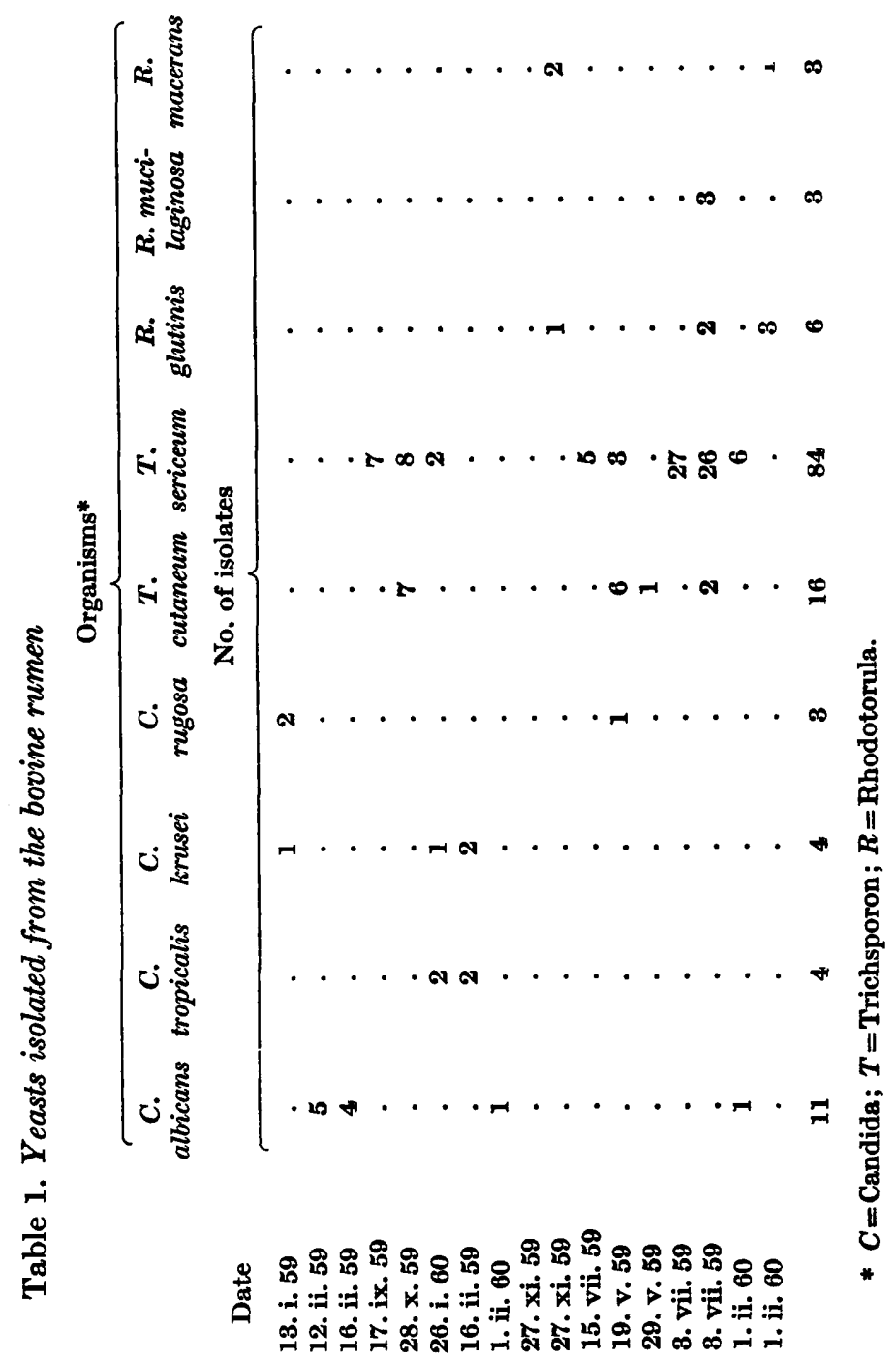

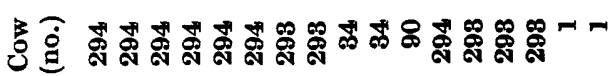

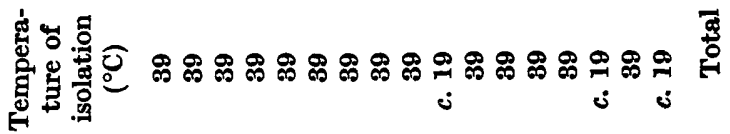

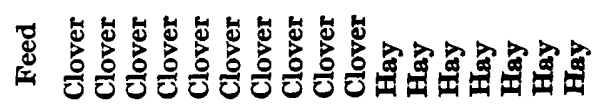


strongly on solid auxanographic medium. Authentic strains of $T$. sericeum and T. capitatum were obtained from the Centraalbureau voor Schimmelcultures and compared with the bovine isolates. As the results from this comparison were inconclusive, it was decided to allocate the bovine isolates to the longer established species, $T$. sericeum.

Rhodotorula spp. Rhodotorula glutinis (Fres.) Harrison, R. mucilaginosa (Jorg.) Harrison and $\boldsymbol{R}$. macerans Frederikson were isolated from three cows. All these isolations were made at room temperature. $\boldsymbol{R}$. glutinis and $\boldsymbol{R}$. macerans were the only yeasts isolated from the non-fistulated cow (34).

\section{Hay and pasture isolates}

Six species belonging to three genera were identified from the twenty-one isolates obtained from hay and pasture samples. All the yeasts recovered from the hay and pasture samples were from cultures incubated at room temperature; none could be isolated at $39^{\circ}$. No yeasts were recovered from the clover samples. Particulars of the species isolated are given below.

Cryptococcus laurentii (Kufferath) Skinner and C. luteolus (Saito) Skinner were recovered from pasture samples.

Torulopsis ingeniosa di Menna was recovered from pasture. Rhodotorula glutinis (Fres.) Harrison was recovered from pasture and both $R$. macerans Frederikson and $\boldsymbol{R}$. mucilaginosa (Jorg.) Harrison were found on hay.

\section{DISCUSSION}

In view of the similarity of the anaerobic fermentations in the bovine rumen and lower intestinal tract, the presence of yeasts in the rumen is not surprising. With the exception of Candida albicans, those yeasts isolated here from cultures incubated at $39^{\circ}$ are represented among those found in the bovine caecum by Van Uden \& do Carmo Sousa (1957), and fall into their second group of yeasts: facultative saprophytes of warm-blooded animals. It is evident that the yeasts isolated here are true rumen inhabitants since yeasts which could grow at $39^{\circ}$ were not isolated from the samples of feed material, and the rumen yeasts were not represented among those considered as part of the normal flora of the leaves of pasture plants (di Menna, 1959). The yeasts isolated here from hay and pasture samples were similar to those found by di Menna (1959). Of these, only Rhodotorula spp. were isolated from the rumen, and then only at room temperature.

The yeasts isolated from the rumen at $39^{\circ}$ fall into two groups, fermenting and non-fermenting yeasts. The presence of either group appears to be dependent upon the diet of the cows. Except for one isolate of Candida albicans all the yeasts isolated from hay-fed cows were of the non-fermenting type. This isolate of $C$. albicans was made from cow no. 1 only a few days after it was returned to a hay diet. All the other fermenting yeasts were recovered from cows fed on fresh clover. This division into fermenting and non-fermenting yeast flora may be dependent on the soluble sugar content of fresh clover and clover hay. The soluble sugar content of fresh red clover may reach as high as $9 \%$ of the weight, calculated on a dry-matter basis, while that of clover hay is usually considerably lower depending on the conditions at the time of harvesting (Dr R. W. Bailey, personal communication). 
The failure to isolate yeasts other than Rhodotorula species from the non-fistulated cow was probably the result of having to use an oesophageal tube for sampling. The samples collected were always small and from an undetermined part of the rumen and reticulum. From experience with fistulated cows it is known that it is difficult to obtain a representative sample of rumen liquor because of layering of the rumen contents.

The numbers of yeasts found in the rumen are too low for them to be important in the provision of soluble fermentation products although they undoubtedly contribute to the total microbial protein available to the animal.

Our thanks are due to Dr N. J. W. Kreger-van Rij (Yeast Division, Centraalbureau voor Schimmelcultures, Delft, Netherlands) for her identification of the Trichosporon cutaneum cultures.

This paper is Soil Bureau Publication, No. 236.

\section{REFERENCES}

Clarke, R. T. J. (1960). Rumen Candida species and bovine mastitis. N.Z. vet. J. 8, 79. Cunningham H. M. \& Brisson, G. J. (1955). Note on alcoholic fermentation in the stomachs of lambs fed high-glucose diets. Canad. J. agric. Sci. 35, 511.

Ingram, M. \& McGaughey, C. A. (1948). Microflora of the rumen of the sheep. Nature, Lond. 162, 533.

Lodder, J. \& Kreger-van Riv, N. J. W. (1952). The Yeasts. A Taxonomic Study. Amsterdam: North Holland Publishing Company.

Lubinsky, G. (1957). Studies on the evolution of the Ophryoscolecidae (Ciliata: Oligotricha). I. A new species of Entodinium with 'caudatum', 'loboso-spinosum', and 'dubardi' forms, and some evolutionary trends in the genus Entodinium. Canad.J. Zool. $35,111$.

LUBINSky, G. (1958). Ophryoscolecidae (Ciliata: Entodiniomorphida) of reindeer (Rangifer tarandus L.) from the Canadian Arctic. I. Entodiniinae. Canad. J. Zool. 36, 819.

Menna, M. E. DI (1957). The isolation of yeasts from soil. J. gen. Microbiol. 17, 678.

Menna, M. E. DI (1959). Yeasts from the leaves of pasture plants. N.Z. J. agric. Res. 2, 394.

Parle, J. N. (1957). Yeasts isolated from the mammalian alimentary tract. J. gen. Microbiol. 17, 363.

van Uden, N. \& Carmo Sousa, L. Do (1957). Yeasts from the bovine caecum. J. gen. Microbiol. 16, 385. 